\title{
The triglycerides and glucose index is associated with cardiovascular risk factors in normal-weight children and adolescents
}

\author{
Luis E. Simental-Mendía ${ }^{1}$, Gabriela Hernández-Ronquillo ${ }^{1}$, Rita Gómez-Díaz ${ }^{2}$, Martha Rodríguez-Morán ${ }^{1}$ and \\ Fernando Guerrero-Romero ${ }^{1}$
}

BACKGROUND: Given the usefulness of the product of triglycerides and glucose (TyG) to recognize individuals at high risk for developing cardiovascular events, the aim of this study was to determine whether the TyG index is associated with the presence of cardiovascular risk factors in apparently healthy normal-weight children and adolescents.

METHODS: Apparently healthy children and adolescents with normal weight, aged 6-15 years, were enrolled in a population-based cross-sectional study. The children were allocated into groups with and without cardiovascular risk factors. Cardiovascular risk factors were considered as the occurrence of at least one of the following: elevated blood pressure, hypertriglyceridemia, low high-density lipoprotein cholesterol (HDL-C), or hyperglycemia.

RESULTS: A total of 2,117 children and adolescents were enrolled in the study; of them, 1,078 (50.9\%) participants exhibited cardiovascular risk. The adjusted logistic regression analysis showed that elevated TyG index was significantly associated with hypertriglyceridemia (odds ratio $(O R)=96.45$, 95\% confidence interval (Cl): 48.44-192.04), low HDL-C $(\mathrm{OR}=2.07,95 \% \mathrm{Cl}: 1.46-2.92)$, and hyperglycemia $(\mathrm{OR}=3.11$, 95\% Cl: 2.05-4.72), but not with elevated blood pressure $(\mathrm{OR}=1.39,95 \% \mathrm{Cl}: 0.89-2.16)$.

CONCLUSION: The elevated TyG index is associated with the presence of cardiovascular risk factors in healthy normalweight children and adolescents.

$\mathbf{P}$ henotype of individuals suspected to be metabolically obese is characterized by the presence of metabolic abnormalities in subjects with normal weight (1); findings that highlight the need to strengthen efforts focused on the early detection of those individuals who, irrespective of obesity, could be at high risk for developing cardiometabolic diseases.

Nonetheless, most health policies are focused on the population with obesity, which represents unequal opportunities for the non-obese population that may have high cardiovascular risk. In this regard, although recognition of cardiovascular risk factors in the metabolically obese, normalweight individuals should be considered as a public health priority, studies that have evaluated this phenotype in the pediatric population are scarce $(2,3)$.

Previously, we have proposed the product of triglycerides and glucose $(\mathrm{TyG})$ as an alternative tool for identifying insulin resistance $(4,5)$, which has been assessed in adults (6-9) and obese adolescents (10) but not in children and youth with normal weight.

A recent study conducted in American children and adolescents, stratified by age and gender, analyzed multiple profiles of lipids, blood pressure, and anthropometry to identify subgroups with cardiovascular risk, showing that systolic and diastolic blood pressures were less discriminating than lipid profile (11).

Given the previously demonstrated usefulness of the TyG index to recognize the risk for developing metabolic diseases in adult population $(12,13)$, the aim of this study was to determine whether the TyG index is associated with the presence of cardiovascular risk factors in apparently healthy normal-weight Mexican children and adolescents.

\section{METHODS}

After the approval of the protocol by the Mexican Social Security Institute Research Committee, in accordance with the ethical principles of the Declaration of Helsinki, a population-based crosssectional study was carried out. A written informed consent was obtained from at least one of each participant's parents along with the informed assent from the children and adolescents who were included in the study.

Eligible participants were apparently healthy normal-weight children and adolescents aged 6-15 years, from the general population of Mexico City, who were recruited in seven primary care units and from one elementary school, and from general population in Durango city.

Children and adolescents were allocated into groups with and without cardiovascular risk factors. In order to control the potential sources of bias related to age, participants were stratified by age into children aged 6-9 years and adolescents aged 10-15 years (14). Obesity, smoking, alcohol intake, pregnancy, previous diagnosis of diabetes, kidney, hepatic, endocrine diseases, intake of drugs, or any

\footnotetext{
'Biomedical Research Unit of the Mexican Social Security Institute at Durango, Durango, México; ${ }^{2}$ Unidad de Investigación Médica en Epidemiología Clínica, UMAE Hospital de Especialidades del CMN Siglo XXI del IMSS, México City, México. Correspondence: Fernando Guerrero-Romero (guerrero.romero@gmail.com) 
kind of medical treatment were exclusion criteria. Sociodemographic characteristics, such as sex, age, smoking, and intake of alcohol or drugs, health status, and eligibility to participate were corroborated through a standardized direct interview, clinical examination, and laboratory tests.

\section{Definitions}

The presence of cardiovascular risk factors was considered as the occurrence of at least one of the following: systolic and/or diastolic blood pressure $\geq 90^{\text {th }}$ percentile according to age, sex, and height (15); triglycerides $\geq 100 \mathrm{mg} / \mathrm{dl}$ (refs 16,17); high-density lipoprotein cholesterol (HDL-C) $\leq 40 \mathrm{mg} / \mathrm{dl}$ (refs 16,17); and fasting glucose $\geq 100 \mathrm{mg} / \mathrm{dl}$ (18).

The cutoff for elevated TyG index was 4.65 and 4.72 in children and adolescents, respectively, which was obtained from an independent cohort (data not published).

Normal weight was defined by body mass index (BMI) between the $15^{\text {th }}$ and $85^{\text {th }}$ percentiles for age and gender (19).

\section{Measurements}

The TyG index was calculated by the following formula: $\operatorname{Ln}$ [fasting triglycerides $(\mathrm{mg} / \mathrm{dl}) \times$ fasting glucose $(\mathrm{mg} / \mathrm{dl})] / 2(4)$.

In the standing position, after $8-10 \mathrm{~h}$ of fasting conditions, the weight and height were measured with participants in light clothing and without shoes. Weight and height were measured using a fixed scale with stadimeter (Tanita TBF-215, Tokyo, Japan). Waist circumference was measured to the nearest centimeter with a flexible steel tape while the participants were in standing position. The two anatomical landmarks used to determine tape placement were midway between the lowest portion of the rib cage and the superior border of the iliac crest (laterally) and the umbilicus (anteriorly). The BMI was calculated as weight (kilograms) divided by height (meters) squared.

Blood pressure was measured with the child seated and according to the recommendations of the Seventh Report of the Joint National Committee on Prevention, Detection, Evaluation, and Treatment of High Blood Pressure (20).

\section{Assays}

A whole-blood sample was collected from an antecubital vein after $8-10 \mathrm{~h}$ of overnight fasting. The fasting period was confirmed by direct and independent interview with both the parents and participants in the study. Serum glucose was measured by the glucose-oxidase method, with an intra-assay variation of $1.8 \%$ and an inter-assay variation of $2.1 \%$. Serum total cholesterol and triglycerides were enzymatically measured using spectrophotometric methods. Serum HDL-C fraction was obtained after precipitation by a phosphotungstic reagent. The intra- and inter-assay coefficients of variation were 1.7 and $2.8 \%$ for total cholesterol, 1.3 and $1.7 \%$ for triglycerides, and 1.1 and $1.7 \%$ for HDL-C. All laboratory measurements were performed using a clinical chemistry autoanalyzer (A15; BioSystems, Barcelona, Spain). Low-density lipoprotein cholesterol was calculated using the Friedewald formula (21).

\section{Statistical Analyses}

Differences between the groups were estimated using the two-tailed unpaired Student's $t$-test for comparison of normally distributed quantitative variables; alternatively, Mann-Whitney $U$-test was used for skewed data and $\chi^{2}$-test for categorical variables.

The association between elevated TyG index (independent variable) and cardiovascular risk factors (dependent variables) was evaluated using multiple logistic regression analysis adjusted by those variables that, in bivariate analysis, showed significant differences.

Data analyses were performed using the statistical package SPSS version 15.0 (SPSS, Chicago, IL). A 95\% confidence interval or $P$ value $<0.05$ defined statistical significance.
Table 1. Characteristics of the study population according to the presence of cardiovascular risk

\begin{tabular}{lccc}
\hline & CVR+ & CVR - & $P$ \\
\hline$N$ & 1,078 & 1,039 & \\
Age, years & $11.0 \pm 2.2$ & $10.7 \pm 2.4$ & 0.001 \\
Girls, $n$ (\%) & $611(56.6)$ & $470(45.2)$ & $<0.001$ \\
Boys, $n$ (\%) & $467(43.4)$ & $569(54.8)$ & $<0.001$ \\
Body mass index, kg/m ${ }^{2}$ & $17.8 \pm 2.3$ & $17.2 \pm 2.2$ & $<0.001$ \\
Waist circumference, cm & $66.7 \pm 8.3$ & $64.4 \pm 8.4$ & $<0.001$ \\
Systolic blood pressure, mm Hg & $102.6 \pm 14.4$ & $94.4 \pm 11.3$ & $<0.001$ \\
Diastolic blood pressure, mm Hg & $64.2 \pm 12.1$ & $57.7 \pm 8.8$ & $<0.001$ \\
Fasting glucose, mg/dl & $89.7 \pm 12.4$ & $85.5 \pm 7.6$ & $<0.001$ \\
Total cholesterol, mg/dl & $162.4 \pm 42.1$ & $166.6 \pm 34.1$ & 0.01 \\
HDL-C, mg/dl & $44.9 \pm 13.6$ & $53.6 \pm 10.2$ & $<0.001$ \\
Triglycerides, mg/dl & $98.7 \pm 48.0$ & $68.7 \pm 18.4$ & $<0.001$ \\
TyG index & $4.48 \pm 0.24$ & $4.31 \pm 0.16$ & $<0.001$ \\
\hline
\end{tabular}

CVR-, without cardiovascular risk; CVR+, with cardiovascular risk; HDL-C, highdensity lipoprotein cholesterol; TyG, product of triglycerides and glucose levels. $n=2,117$.

\section{RESULTS}

A total of 2,117 children and adolescents with an average age of $10.8 \pm 2.3$ years, 1,081 (51.0\%) girls and 1,036 (49.0\%) boys, were enrolled in the study. Of these, 1,078 (50.9\%) exhibited cardiovascular risk: 328 (15.4\%) elevated blood pressure, 388 (18.3\%) hypertriglyceridemia, 529 (24.9\%) low HDL-C, and $240(11.3 \%)$ hyperglycemia.

Participants with cardiovascular risk had higher age, BMI, waist circumference, total cholesterol levels, and TyG index compared with those without cardiovascular risk (Table 1).

The girls with cardiovascular risk showed lower glucose concentrations and higher triglyceride levels and TyG index than boys, whereas in the group with no cardiovascular risk, the girls exhibited higher age, BMI, systolic and diastolic blood pressures, and triglyceride levels than the boys (Table 2).

An additional analysis stratifying population according to age was performed. The children (6-9 years) with cardiovascular risk had higher BMI, waist circumference, systolic and diastolic blood pressures, glucose, triglyceride levels, and TyG index as well as lower total cholesterol and HDL-C concentrations than children with no cardiovascular risk (Table 3).

Regarding adolescents (participants aged 10-15 years), those with cardiovascular risk showed higher BMI, waist circumference, systolic and diastolic blood pressure, glucose, triglyceride levels, and TyG index, as well as lower HDL-C concentration, compared with the adolescents without cardiovascular risk. Nonetheless, there were no significant differences in total cholesterol levels between adolescents with and without cardiovascular risk (Table 3 ).

Table 4 shows distribution of the target population according to TyG index quintiles. Children and adolescents 


\section{Articles $\mid$ simental-Mendia et al.}

Table 2. Characteristics of the study population according to sex

\begin{tabular}{|c|c|c|c|c|c|c|}
\hline & \multicolumn{2}{|c|}{ CVR+ } & \multirow[t]{2}{*}{$P$} & \multicolumn{2}{|c|}{ CVR - } & \multirow[t]{2}{*}{$P$} \\
\hline & Girls & Boys & & Girls & Boys & \\
\hline$N$ & 611 & 467 & & 470 & 569 & \\
\hline Age, years & $11.0 \pm 2.2$ & $11.1 \pm 2.2$ & 0.401 & $10.9 \pm 2.4$ & $10.5 \pm 2.3$ & 0.005 \\
\hline Body mass index, $\mathrm{kg} / \mathrm{m}^{2}$ & $17.8 \pm 2.4$ & $17.7 \pm 2.1$ & 0.434 & $17.5 \pm 2.4$ & $16.9 \pm 2.1$ & $<0.001$ \\
\hline Waist circumference, $\mathrm{cm}$ & $66.9 \pm 8.5$ & $66.4 \pm 8.1$ & 0.340 & $64.9 \pm 8.3$ & $64.0 \pm 8.5$ & 0.101 \\
\hline Systolic blood pressure, $\mathrm{mmHg}$ & $102.6 \pm 14.1$ & $102.5 \pm 14.8$ & 0.930 & $96.2 \pm 11.2$ & $93.0 \pm 11.3$ & $<0.001$ \\
\hline Diastolic blood pressure, $\mathrm{mmHg}$ & $64.3 \pm 11.8$ & $64.1 \pm 12.4$ & 0.799 & $58.8 \pm 8.7$ & $56.7 \pm 8.8$ & $<0.001$ \\
\hline Fasting glucose, mg/dl & $88.8 \pm 12.1$ & $90.9 \pm 12.6$ & 0.007 & $85.2 \pm 7.9$ & $85.8 \pm 7.3$ & 0.240 \\
\hline Total cholesterol, mg/dl & $161.9 \pm 39.7$ & $162.9 \pm 45.0$ & 0.701 & $165.3 \pm 34.9$ & $167.6 \pm 33.5$ & 0.283 \\
\hline HDL cholesterol, mg/dl & $44.3 \pm 13.2$ & $45.6 \pm 14.1$ & 0.145 & $53.3 \pm 10.7$ & $53.8 \pm 9.7$ & 0.402 \\
\hline Triglycerides, mg/dl & $102.5 \pm 48.6$ & $93.6 \pm 46.8$ & $<0.001^{\mathrm{a}}$ & $70.1 \pm 18.7$ & $67.5 \pm 18.1$ & $0.03^{a}$ \\
\hline TyG index & $4.50 \pm 0.23$ & $4.46 \pm 0.25$ & 0.009 & $4.32 \pm 0.16$ & $4.30 \pm 0.16$ & 0.124 \\
\hline
\end{tabular}

CVR -, without cardiovascular risk; CVR+, with cardiovascular risk; HDL-C, high-density lipoprotein cholesterol; TyG, product of triglycerides and glucose levels.

a $P$ value estimated with the Mann-Whitney U-test.

$n=2,117$.

Table 3. Characteristics of the study population stratified by age

\begin{tabular}{|c|c|c|c|c|c|c|}
\hline & \multicolumn{2}{|c|}{ Children (6-9 years) } & \multirow[t]{2}{*}{$P$} & \multicolumn{2}{|c|}{ Adolescents (10-15 years) } & \multirow[t]{2}{*}{$P$} \\
\hline & CVR+ & CVR - & & CVR+ & CVR- & \\
\hline$N$ & 275 & 340 & & 803 & 699 & \\
\hline Girls, $n(\%)$ & $164(59.6)$ & $143(42.0)$ & $<0.001$ & $447(55.6)$ & $327(46.7)$ & $<0.001$ \\
\hline Body mass index, $\mathrm{kg} / \mathrm{m}^{2}$ & $15.8 \pm 1.4$ & $15.6 \pm 1.3$ & 0.01 & $18.5 \pm 2.1$ & $18.0 \pm 2.2$ & $<0.001$ \\
\hline Diastolic blood pressure, $\mathrm{mm} \mathrm{Hg}$ & $63.2 \pm 12.6$ & $55.5 \pm 8.5$ & $<0.001$ & $64.5 \pm 11.9$ & $58.7 \pm 8.8$ & $<0.001$ \\
\hline Fasting glucose, mg/dl & $86.2 \pm 11.6$ & $84.3 \pm 7.3$ & 0.02 & $90.9 \pm 12.4$ & $86.1 \pm 7.7$ & $<0.001$ \\
\hline Total cholesterol, mg/dl & $152.8 \pm 32.2$ & $166.1 \pm 27.2$ & $<0.001$ & $165.6 \pm 44.5$ & $166.8 \pm 37.0$ & 0.575 \\
\hline $\mathrm{HDL}-\mathrm{C}, \mathrm{mg} / \mathrm{dl}$ & $42.1 \pm 11.7$ & $53.5 \pm 9.7$ & $<0.001$ & $45.8 \pm 14.1$ & $53.6 \pm 10.4$ & $<0.001$ \\
\hline
\end{tabular}

CVR -, without cardiovascular risk; CVR+, with cardiovascular risk; HDL-C, high-density lipoprotein cholesterol; TyG, product of triglycerides and glucose levels.

a $P$ value estimated with the Mann-Whitney U-test.

$n=2,117$.

in the highest quintile exhibited higher glucose and triglyceride levels but lower HDL-C concentrations compared with the lower quintiles. In addition, participants in the highest quintile showed a significantly higher frequency of low HDL-C and cardiovascular risk factors than those in the lower quintiles. Besides, elevated TyG index was found in 240 (11.3\%), 45 (7.3\%), and 195 (12.9\%) participants in the overall population, children, and adolescents, respectively.

The simple logistic regression analysis, comparing the highest quintile vs. the lowest quintile of TyG index, showed a significant association between elevated TyG index with hypertriglyceridemia, low HDL-C, and hyperglycemia, but not with elevated blood pressure. In the adjusted multiple logistic regression analysis, the elevated TyG index remained significantly associated with hypertriglyceridemia, low HDL$\mathrm{C}$, and hyperglycemia, but not with elevated blood pressure (Table 5).

\section{DISCUSSION}

The findings of the present study suggest that the TyG index is significantly associated with the presence of cardiovascular risk factors in apparently healthy children and adolescents with normal weight.

Currently, studies about cardiovascular risk factors associated with the metabolically obese normal-weight children and adolescents are scarce and inconsistent. Our results agree with a meta-analysis that reported an increased risk for cardiovascular disease in adults who exhibited an abnormal 
Table 4. Characteristics of the study population according to TyG index quintiles

\begin{tabular}{|c|c|c|c|c|c|}
\hline & \multicolumn{5}{|c|}{ TyG index quintiles } \\
\hline & Low & $2^{\text {nd }}$ & $3^{r d}$ & $4^{\text {th }}$ & High \\
\hline$N$ & 435 & 450 & 409 & 415 & 408 \\
\hline Age, years & $10.9 \pm 2.6$ & $10.5 \pm 2.3$ & $10.8 \pm 2.3$ & $10.8 \pm 2.2$ & $11.2 \pm 1.9$ \\
\hline Girls, $n(\%)$ & $194(44.5)$ & $204(45.3)$ & $205(50.1)$ & $241(58.0)$ & $237(58.0)$ \\
\hline Body mass index, $\mathrm{kg} / \mathrm{m}^{2}$ & $17.3 \pm 2.3$ & $17.0 \pm 2.2$ & $17.3 \pm 2.2$ & $17.8 \pm 2.2$ & $18.1 \pm 2.2$ \\
\hline Waist circumference, cm & $65.0 \pm 8.6$ & $64.0 \pm 8.1$ & $65.0 \pm 8.1$ & $66.1 \pm 8.9$ & $67.7 \pm 8.1$ \\
\hline Systolic blood pressure, $\mathrm{mm} \mathrm{Hg}$ & $97.9 \pm 13.5$ & $96.1 \pm 14.1$ & $98.3 \pm 13.3$ & $100.0 \pm 13.3$ & $100.7 \pm 13.4$ \\
\hline Diastolic blood pressure, $\mathrm{mm} \mathrm{Hg}$ & $60.3 \pm 11.3$ & $59.3 \pm 10.8$ & $60.9 \pm 10.4$ & $62.0 \pm 11.4$ & $62.7 \pm 11.2$ \\
\hline Elevated blood pressure, $n(\%)$ & $69(15.8)$ & $49(10.8)$ & $63(15.4)$ & $72(17.3)$ & $75(18.3)$ \\
\hline Fasting glucose, mg/dl & $83.8 \pm 11.0$ & $85.0 \pm 8.8$ & $87.6 \pm 8.2$ & $89.7 \pm 9.5$ & $92.8 \pm 12.1^{*}$ \\
\hline Hyperglycemia, $n$ (\%) & $33(7.5)$ & $28(6.2)$ & $31(7.5)$ & $54(13.0)$ & $93(22.7)$ \\
\hline $\mathrm{HDL}-\mathrm{C}, \mathrm{mg} / \mathrm{dl}$ & $51.7 \pm 13.2$ & $50.6 \pm 12.2$ & $49.0 \pm 11.9$ & $48.2 \pm 12.6$ & $46.0 \pm 13.4^{*}$ \\
\hline Low HDL-C, $n$ (\%) & 78 (17.9) & $95(21.1)$ & $90(22.0)$ & $106(25.5)$ & $159(38.9)^{*}$ \\
\hline Triglycerides, mg/dl & $45.8 \pm 10.9$ & $64.8 \pm 7.3$ & $75.9 \pm 8.4$ & $93.6 \pm 12.1$ & $144.1 \pm 44.6^{*}$ \\
\hline Hypertriglyceridemia, $n$ (\%) & $0(0.0)$ & $0(0.0)$ & $0(0.0)$ & $42(10.1)$ & $345(84.5)$ \\
\hline CVR, $n(\%)$ & $161(37.0)$ & $151(33.5)$ & $157(38.3)$ & $214(51.5)$ & $395(96.8)^{*}$ \\
\hline
\end{tabular}

CVR, cardiovascular risk; HDL-C, high-density lipoprotein cholesterol; TyG, product of triglycerides and glucose levels.

${ }^{*} P<0.05$.

$n=2,117$.

Table 5. Logistic regression analysis that evaluates the association between elevated TyG index (independent variable) and cardiovascular risk factors (dependent variables) comparing the highest quintile vs. the lowest quintile of TyG index

\begin{tabular}{|c|c|c|c|c|c|c|}
\hline & \multicolumn{3}{|c|}{ Not adjusted } & \multicolumn{3}{|c|}{ Adjusted $^{a}$} \\
\hline & OR & $95 \% \mathrm{Cl}$ & $P$ & OR & $95 \% \mathrm{Cl}$ & $P$ \\
\hline Elevated blood pressure (SBP to DBP $\geq 90$ percentile) & 1.26 & $0.86-1.86$ & 0.22 & 1.39 & $0.89-2.16$ & 0.14 \\
\hline Low HDL-C( $\leq 40 \mathrm{mg} / \mathrm{dl})$ & 2.18 & $1.58-3.00$ & $<0.001$ & 2.07 & $1.46-2.92$ & $<0.001$ \\
\hline
\end{tabular}

DBP, diastolic blood pressure; OR, odds ratio; SBP, systolic blood pressure; TyG, product of triglycerides and glucose.

${ }^{a}$ Analysis adjusted by age, sex, body mass index, and waist circumference.

metabolic status with normal weight (22). However, studies in the pediatric population evaluating the association between the metabolically obese normal-weight children and cardiovascular disease risk are scarce (23).

Insulin resistance is probably the main underlying characteristic of the metabolic disorders in metabolically obese normal-weight individuals (24-27), making this characteristic a priority for public health policy decisions in these individuals. Nonetheless, given that the hyperinsulinemiceuglycemic clamp (the gold standard test) is expensive, complicated, invasive, and not available in clinical setting, its use in large-scale epidemiological studies is not feasible. For this reason, the development of simple, accessible, and reliable methods for measurement of insulin sensitivity emerges as a need for the clinical setting.

In this regard, the TyG index, based on routine laboratory measurements, has the advantage of not requiring insulin determination and of being a simple, reproducible, and reliable tool to identify the presence of insulin resistance $(14,28-31)$. Our results show that the TyG index may be a biomarker of cardiovascular risk in children and adolescents.

Given that the TyG index is calculated based on fasting triglyceride and glucose levels, the strong association of the TyG index with both hypertriglyceridemia and hyperglycemia was an expected finding. Moreover, regarding the association between TyG index and hyperglycemia, our findings are consistent with the report by Mohd et al. in obese adolescents (12).

The TyG index was significantly associated with low HDL-C, the most prevalent (24.9\%) metabolic disturbance in the target population of our study, which is an independent risk factor for the development of atherosclerosis in children (32). To the best of our knowledge, the possible association between TyG index and low HDL-C has not been previously evaluated. Undoubtedly, further studies in children are needed to corroborate our findings.

Regarding blood pressure, the TyG index showed a significant association with both systolic and diastolic blood pressure. In this regard, in adult population, it has been 


\section{Articles | Simental-Mendía et al.}

reported that, independent of adiposity, the TyG index is strongly associated with incident hypertension (33), and might be useful for early identification of individuals at a high risk of developing a cardiovascular event, as supported by a study by Sánchez-Iñigo et al. (34).

It is important to note that at least one metabolic disorder was found in half of the target population in our study, which indicates a high prevalence of cardiovascular risk factors in children and adolescents without obesity. This finding emphasizes the fact that current health policies, focused on obese population, do not recognize a high proportion of children and adolescents with cardiovascular risk factors. In this context, our results reveal an emerging health problem that requires preventive interventions by corresponding health authorities, which should be oriented toward the search for cardiovascular risk factors such as hypertriglyceridemia, low HDL-C, and hyperglycemia in order to identify normal-weight children and adolescents at high risk for cardiovascular disease.

Several limitations of our study deserve to be mentioned. First, the cross-sectional design of the study does not allow establishing with certainty the temporal relationship between the independent and dependent variables. Second, it was not possible to stratify the population according to pubertal status owing to the lack of Tanner stage data; nevertheless, given the aim of the study, this limitation did not significantly influence our conclusions. Third, physical activity was not assessed; however, children and adolescents of our target population are characterized by a sedentary life style, which minimizes a possible source of bias. Finally, given that the TyG index is calculated using triglyceride and glucose levels, it was expected a significant association between elevated TyG index with hypertriglyceridemia and hyperglycemia; nonetheless, it is necessary to keep in mind that elevated triglyceride and glucose concentrations as well as the TyG index have been independently associated with the development of cardiovascular disease (34-36).

In conclusion, the results of our study show that the elevated TyG index is significantly associated with the presence of cardiovascular risk factors in normal-weight healthy children and adolescents. However, future clinical and epidemiological studies are required in this field in order to confirm our results because the evidence is still limited and the use of TyG index could provide a useful tool for early detection of cardiovascular risk factors in pediatric population irrespective of obesity.

Disclosure: The authors declare no conflict of interest.

\section{REFERENCES}

1. Lee SH, Ha HS, Park YJ, et al. Identifying metabolically obese but normalweight (MONW) individuals in a nondiabetic Korean population: the Chungju Metabolic disease Cohort (CMC) study. Clin Endocrinol 2011;75:475-81.

2. Kelishadi R, Cook SR, Motlagh ME, et al. Metabolically obese normal weight and phenotypically obese metabolically normal youths: the CASPIAN Study. J Am Diet Assoc 2008;108:82-90.
3. Pacifico L, Poggiogalle E, Costantino F, et al. Acylated and nonacylated ghrelin levels and their associations with insulin resistance in obese and normal weight children with metabolic syndrome. Eur J Endocrinol 2009;161:861-70.

4. Simental-Mendía LE, Rodríguez-Morán M, Guerrero-Romero F. The product of fasting glucose and triglycerides as surrogate for identifying insulin resistance in apparently healthy subjects. Metab Syndr Relat Disord 2008;6:299-304.

5. Guerrero-Romero F, Simental-Mendía LE, González-Ortiz M, et al. The product of triglycerides and glucose, a simple measure of insulin sensitivity. Comparison with the euglycemic-hyperinsulinemic clamp. J Clin Endocrinol Metab 2010;95:3347-51.

6. Gastaldelli A, Folli F, DeFronzo RA. The product of triglycerides and glucose as index of insulin resistance. Validation in the SAM study. J Clin Endocrinol Metab 2010;95:3351.

7. Du T, Yuan G, Zhang M, Zhou X, Sun X, Yu X. Clinical usefulness of lipid ratios, visceral adiposity indicators, and the triglycerides and glucose index as risk markers of insulin resistance. Cardiovasc Diabetol 2014; 13:146.

8. Espinel-Bermúdez MC, Robles-Cervantes JA, del Sagrario VillarrealHernández L, et al. Insulin resistance in adult primary care patients with a surrogate index, Guadalajara, Mexico, 2012. J Invest Med 2015;63:247-50.

9. Lee SH, Kwon HS, Park YM, et al. Identifying subgroups of obesity using the product of triglycerides and glucose: the Korea National Health and Nutrition Examination Survey, 2008-2010. Clin Endocrinol 2015;82: 213-20.

10. Mohd Nor NS, Lee S, Bacha F, Tfayli H, Arslanian S. Triglyceride glucose index as a surrogate measure of insulin sensitivity in obese adolescents with normoglycemia, prediabetes, and type 2 diabetes mellitus: comparison with the hyperinsulinemic-euglycemic clamp. Pediatr Diabetes 2016;17:458-65.

11. Ip EH, Leng X, Zhang Q, et al. Risk profiles of lipids, blood pressure, and anthropometric measures in childhood and adolescence: project heartBeat!. BMC Obes 2016;3:9.

12. Irace C, Carallo C, Scavelli FB, et al. Markers of insulin resistance and carotid atherosclerosis. A comparison of the homeostasis model assessment and triglyceride glucose index. Int J Clin Pract 2013;67:665-72.

13. Lee SH, Kwon HS, Park YM, et al. Predicting the development of diabetes using the product of triglycerides and glucose: The Chungju Metabolic Disease Cohort (CMC) StudyPLoS ONE 2014;9:e90430.

14. Guerrero-Romero F, Rodríguez-Morán M, Hernández-Ronquillo G. Network of childhood obesity of the Mexican Social Security Institute. Low serum magnesium levels and its association with high blood pressure in children. J Pediatr 2016;168:e1.

15. National High Blood Pressure Education Program Working Group on High Blood Pressure in Children and Adolescents. The fourth report on the diagnosis, evaluation, and treatment of high blood pressure in children and adolescents. Pediatrics 2004;114 (2 Suppl 4th Report): 555-76.

16. Lee S, Bacha F, Gungor N, Arslanian S. Comparison of different definitions of pediatric metabolic syndrome: relation to abdominal adiposity, insulin resistance, adiponectin, and inflammatory biomarkers. J Pediatr 2008;152:177-84.

17. Cook S, WeitzmanM, Auinger P, NguyenM, Dietz WH. Prevalence of a metabolic syndrome phenotype in adolescents: findings from the third National Health and Nutrition Examination Survey, 1988-1994. Arch Pediatr Adolesc Med 2003;157:821-7.

18. Zimmet P, Alberti KG, Kaufman F, et al. The metabolic syndrome in children and adolescents - an IDF consensus report. Pediatr Diabetes 2007;8:299-306.

19. Ogden CL, Kuczmarski RJ, Flegal KM, et al. Centers for disease control and prevention 2000 growth charts for the United States: improvements to the 1977 National Center for Health Statistics version. Pediatrics 2002;109:45-60.

20. Chobanian AV, Bakris GL, Black HR, et al. Joint National Committee on Prevention, Detection, Evaluation, and Treatment of High Blood 
Pressure, National Heart, Lung, and Blood Institute, National High Blood Pressure Education Program Coordinating Committee. Seventh Report of the Joint National Committee on Prevention, Detection, Evaluation, and Treatment of High Blood Pressure. Hypertension 2003;42:1206-52.

21. Friedewald WT, Levy RI, Fredrickson DS. Estimation of the concentration of low-density lipoprotein cholesterol in plasma, without use of the preparative ultracentrifuge. Clin Chem 1972;18:499-502.

22. Kramer CK, Zinman B, Retnakaran R. Are metabolically healthy overweight and obesity benign conditions? A systematic review and meta-analysis. Ann Intern Med 2013;159:758-69.

23. Guerrero-Romero F, Aradillas-García C, Simental-Mendía LE, et al. Biochemical characteristics and risk factors for insulin resistance at different levels of obesity. Pediatrics 2013;131:e1211-7.

24. Ruderman N, Chisholm D, Pi-Sunyer X, Schneider S. The metabolically obese, normal-weight individual revisited. Diabetes 1998;47: 699-713.

25. Ruderman NB, Berchtold P, Schneider S. Obesity-associated disorders in normal-weight individuals: some speculations. Int J Obes 1982;6(Suppl 1): $151-7$.

26. Ruderman NB, Schneider SH, Berchtold P. The "metabolically-obese," normal-weight individual. Am J Clin Nutr 1981;34:1617-21.

27. St-Onge MP, Janssen I, Heymsfield SB. Metabolic syndrome in normalweight Americans: new definition of the metabolically obese, normalweight individual. Diabetes Care 2004;27:2222-8.

28. Unger G, Benozzi SF, Perruzza F, Pennacchiotti GL. Triglycerides and glucose index: a useful indicator of insulin resistance. Endocrinol Nutr 2014;61:533-40.
29. Lucatello F, Vigna L, Carugno M, Tirelli AS, Bertazzi PA, Riboldi L. Comparison of indexes for assessing insulin resistance for the health surveillance among workers. G Ital Med Lav Ergon 2012;34 (Suppl 3): 748-9.

30. Vasques AC, Novaes FS, de Oliveira Mda S, et al. TyG index performs better than HOMA in a Brazilian population: a hyperglycemic clamp validated study. Diabetes Res Clin Pract 2011;93:e98-100.

31. Abbasi F, Reaven GM. Comparison of two methods using plasma triglyceride concentration as a surrogate estimate of insulin action in nondiabetic subjects: triglycerides $\times$ glucose versus triglyceride/highdensity lipoprotein cholesterol. Metabolism 2011;60:1673-6.

32. Berenson GS, Srinivasan SR, Bao W, Newman WP 3rd, Tracy RE, Wattigney WA. Association between multiple cardiovascular risk factors and atherosclerosis in children and young adults. The Bogalusa Heart Study. N Engl J Med 1998;338:1650-6.

33. Sánchez-Î̃ingo L, Navarro-González D, Pastrana-Delgado J, FernándezMontero A, Martínez JA. Association of triglycerides and new lipid markers with the incidence of hypertension in a Spanish cohort. J Hypertens 2016;34:1257-65.

34. Sánchez-Î́nigo L, Navarro-González D, Fernández-Montero A, PastranaDelgado J, Martínez JA. The TyG index may predict the development of cardiovascular events. Eur J Clin Invest 2016;46:189-97.

35. Nordestgaard BG, Varbo A. Triglycerides and cardiovascular disease. Lancet 2014;384:626-35.

36. Ford ES, Zhao G, Li C. Pre-diabetes and the risk for cardiovascular disease: a systematic review of the evidence. J Am Coll Cardiol 2010;55: $1310-7$. 